\title{
The GoSam package: an overview
}

\author{
Francesco Tramontano*† \\ "CERN" \\ E-mail: Francesco.Tramontano@cern.ch
}

The public code GOSAM for the computation of the one loop virtual corrections to scattering amplitudes in the Standard Model and beyond is presented. Particular emphasis is devoted to the interface with other public tools via the Binoth Les Houches Accord. We show with examples that doing LHC phenomenology including automatically Next to Leading Order QCD corrections is now handy.

"Loops and Legs in Quantum Field Theory" 11th DESY Workshop on Elementary Particle Physics April 15-20, 2012

Wernigerode, Germany

* Speaker.

$\dagger$ On behalf of the GOSAM collaboration: G. Cullen, N. Greiner, G. Heinrich, G. Luisoni, P. Mastrolia, G. Ossola, T. Reiter, F. Tramontano, H. van Deurzen, J.F.G. von Soden-Fraunhofen, E. Mirabella, T. Peraro, J. Reichel, M. Rodgers, J. Schlenk. 


\section{Introduction}

The recent discovery of a new resonance at the Large Hadron Collider (LHC) at CERN with mass $125 \mathrm{GeV}$ [1] is the result of the efforts in the construction and operation of the accelerator and of the detectors as well as of the efficiency in the implementation and interpretation of very detailed analyses on the large amount of informations collected. Now the target is to perform detailed measurements of all the branching ratios of the novel particle while searching for further new resonances in all the accessible mass regions. The first question is to establish if the new resonance is or not the Higgs boson. In this scenario the help of automated tools for the simulation of signals and backgrounds coming from the Standard Model or beyond is desirable and is now possible including Quantum Chromo Dynamic (QCD) corrections up to the Next to Leading Order (NLO) accuracy in the strong coupling constant. The QCD at Hadron Colliders play of course the main role and several fully automated tools exist that include the leading order matrix element, the leading logarithmic correction as a parton shower and the subsequent hadronization, leading to full event simulation [2]. The NLO hard QCD corrections, being in general quite sizable, also have to be included to get predictions with a theoretical error comparable with the accuracy attainable at the LHC. NLO QCD corrections have been successfully matched in full generality with a parton shower avoiding the double counting that derive by the fact that the shower produces also some of the emission predicted in the full NLO calculations [3]. In this way a number of processes have been studied at NLO accuracy plus parton shower. The deep understanding of fundamental properties and the structure of the divergences of QCD corrections up to the NLO made the automation of the subtractions of the divergences and the matching to the parton shower feasible in full generality. On the other hand progress has been done in the computation of the virtual part of the NLO correction exploiting the analytic properties of loop amplitudes. Advanced computer languages and the increasing cpu performances give now the possibility to automatize also the computation of the virtual corrections even for complicated hard processes, i.e. processes with many legs in the final state.

A key ingredient for the full automation of NLO computation is given by the Binoth Les Houches Accord [4] (BLHA) that fixes a set of universal rules for the communication among different programs that can provide the complementary ingredients needed for the full event simulation.

It is the aim of this presentation to illustrate the usage of GOSAM [5], a new fully automated package for the computation of the one loop matrix elements, and its interfacing with other programs for the integration over the phase space of both the real and the virtual corrections to Born processes.

\section{GOSAM}

GOSAM is a python package that has been designed for the generation of codes for the numerical evaluation of one loop amplitudes. At the moment the code produced are in Fortran95. Inside GOSAM a number of libraries are used: the QGRAF [6] Fortran library is used for the generation of the Feynman diagrams, while FORM [7] is used for the algebraic treatment of the diagrams mainly through the SPINNEY [8] FORM package, the formulas are then optimized and written by the java package HAGGIES [9], finally once a code for a process has been generated it runs calling libraries for the reduction of tensor integrals and scalar integral evaluation [10]. 
The construction of the codes has a very simple structure, the aim is to write efficiently the numerator of each Feynman diagram (or a group of them) as a multivariate polynomial of the components of the loop momentum. For each physical phase space point such polynomial in the loop momentum gets numerical coefficients related to the specific kinematic configuration and as a tensor integrand sitting on a certain set of Feynman denominators is reduced and computed numerically by dedicated libraries.

After several years since the seminal work of Passarino and Veltman [11], loop tensor integral reduction techniques have seen new progress. The original algorithm has been extended to optimize the numerical evaluation of the form factors especially near unstable configurations [12]. On the other hand, the parametric form of the residues of the multipole expansion of the Feynman integrands has been fully determined and exploited to construct recursive algorithms based on polynomial interpolation, leading to the direct numerical determination of the coefficients of the scalar integral [13].

In general two factors determine which method is the fastest or more efficient: the efficiency of the tensor reduction library and the way the numerator is prepared and matched with the tensor reduction library. Further, having more then one construction leads to valuable consistency check on the results. Alternative methods for the construction of the numerator function to match with loop integrals have also been proposed in [14]. A schematic representation of the functionality of the codes generated by GOSAM is given in Figure 1. The basic tensor reduction implemented is

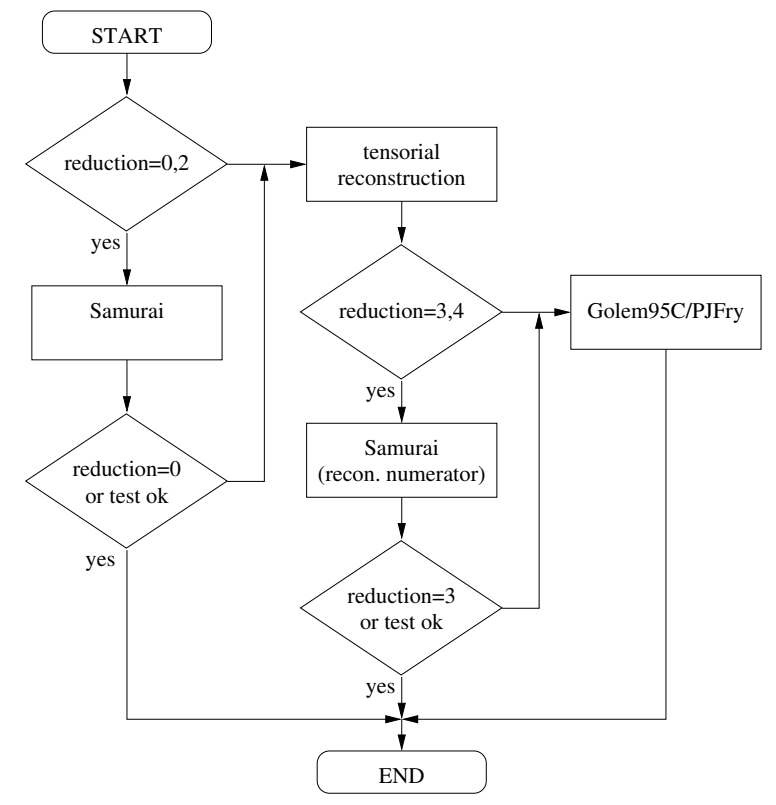

Figure 1: Reduction flow of the GOSAM codes for the evaluation of the numerical virtual amplitudes.

through the SAMURAI library, based on the integrand reduction method mentioned above, that also provides tests to establish the quality of the result. If the test is not passed then the reduction of the tensor integrals is repeated with GOLEM95. For these cases the codes are equipped with a second tensorial representation for the numerator and give more accurate results while remaining in double precision. The reduction through SAMURAI is the default because it turns out to be a 
Table 1: Sample of processes for which GOSAM has been compared to the literature.

\begin{tabular}{|ll|}
\hline process & process \\
\hline$e^{+} e^{-} \rightarrow u \bar{u}$ & $p p \rightarrow W^{ \pm} j j$ \\
$e^{+} e^{-} \rightarrow t \bar{t}$ & $p p \rightarrow W^{ \pm} b \bar{b}$ (massive b's) \\
$u \bar{u} \rightarrow d \bar{d}$ & $e^{+} e^{-} \rightarrow e^{+} e^{-} \gamma$ (QED) \\
$g g \rightarrow g g$ & $p p \rightarrow t \bar{t} H$ \\
$g g \rightarrow g Z$ & $p p \rightarrow t \bar{t} Z$ \\
$p p \rightarrow t \bar{t}$ & $\gamma \gamma \rightarrow \gamma \gamma \gamma \gamma$ (fermion loop) \\
$b g \rightarrow H b$ & $p p \rightarrow W^{+} W^{+} j j$ \\
$\gamma \gamma \rightarrow \gamma \gamma(\mathrm{W}$ loop) & $p p \rightarrow b \bar{b} b \bar{b}$ \\
$p p \rightarrow W^{ \pm} j$ (QCD corr.) & $p p \rightarrow W^{+} W^{-} b \bar{b}$ \\
$p p \rightarrow W^{ \pm} j$ (EW corr.) & $p p \rightarrow t \bar{t} b \bar{b}$ \\
$p p \rightarrow W^{ \pm} t$ & $u \bar{d} \rightarrow W^{+} g g g$ \\
\hline
\end{tabular}

bit faster then the one through GOLEM95.

This switch happens dynamically during the computation of the virtual matrix element, on a diagram by diagram (or group of diagrams) base. Finally, for QCD corrections, the renormalised result for the single pole is compared to the expectation given by the well known formulas expressing the universal behaviour of the infrared QCD divergences, and if the agreement do not fulfil the required accuracy the whole amplitude is computed again using GOLEM95. We have not performed a full study of the correlation among the numerical instabilities of our procedures and the relative importance of the virtual correction. Experimentally we find that fixing the threshold for the mismatch of the single pole as

$$
\varepsilon=\frac{\text { Single pole }(\text { Exact })-\text { Single pole }(\text { Numerical })}{\frac{\alpha_{s}}{2 \pi} \text { Born }} \lesssim 10^{-4}
$$

has two consequences: first we note that only a very small fraction of points turns out to be above threshold (typically one per million for two to three processes) and, second, the results for the distributions turn out to be in excellent agreement with the one obtained with codes containing analytic formulas for the virtual amplitudes like the MCFM [15] suite of programs. Finally, if this higher level instability trigger finds a phase space point to be still unstable this can be saved on a file to be reprocessed with higher precision with the same GOSAM code that can be compiled even with intermediate or quadruple numerical precision.

A number of pointwise comparisons has been performed with results obtained mainly with other codes or by coding amplitudes reported into the appendix of the relative papers, or simply comparing the numerical results reported for benchmark phase space points. A sample of the comparisons is given in Table 1, while in Table 2 a list of timings is reported for the generation of the codes and the relative running time per phase space point.

We generated codes for the NLO QCD corrections for the production at hadron colliders of four bottom quarks [16] and for the associated production of two $W$ bosons with opposite charge plus two jets [17]. Both computations allowed to test the packages on amplitudes consisting of roughly one thousand diagrams. The running time per phase space point for such matrix elements is around 


\begin{tabular}{|l|r|r|}
\hline Process & Generation [s] & Evaluation [ms] \\
\hline$b g \rightarrow H b$ & 236 & 2.49 \\
$d \bar{d} \rightarrow t \bar{t}$ & 324 & 4.05 \\
$d g \rightarrow d g$ & 398 & 3.08 \\
$e^{+} e^{-} \rightarrow t \bar{t}$ (LanHEP) & 180 & 1.27 \\
$e^{+} e^{-} \rightarrow u \bar{u}$ (AutoTools) & 173 & 0.64 \\
$g g \rightarrow g g$ (LanHep) & 1022 & 1.70 \\
$g g \rightarrow g Z$ & 529 & 15.18 \\
$g g \rightarrow t \bar{t}(\mathrm{UFO})$ & 1225 & 29.45 \\
$H \rightarrow \gamma \gamma$ & 140 & 0.24 \\
$g b \rightarrow e^{-} \bar{v}_{e} t$ & 337 & 2.89 \\
$u \bar{d} \rightarrow e^{-} \bar{v}_{e}$ & 71 & 0.09 \\
$u \bar{d} \rightarrow e^{-} \bar{v}_{e} g$ & 154 & 1.15 \\
$u \bar{u} \rightarrow d \bar{d}$ & 186 & 2.06 \\
$\bar{u} d \rightarrow W^{+} W^{+} \bar{c} s$ & 1295 & 17.37 \\
$\gamma \gamma \rightarrow \gamma \gamma$ & 597 & 6.08 \\
\hline
\end{tabular}

Table 2: Time required for code generation and calculation of one phase-space point. The results were obtained with an Intel(R) Core(TM) i7 CPU 950 @ 3.07GHz. The time for the evaluation of a phase space point is taken as the average of the time obtained from the evaluation of 100 random points, where the code was compiled using gfortran without any optimisation options. The generation of the $R_{2}$ term was set to explicit.

one second for a single subprocess preventing the direct integration on a single core of the full virtual matrix elements. In these cases we proceeded through the re-weighting of a previously obtained sample of Born level un-weighted events to obtain a reasonable estimation of the virtual correction. The integration of the real part and of the subtractions has been performed with the packages MadGraph [18], MadDipole [19] and MadEvent [20]. The gluon initiated four bottom quarks production virtual amplitudes were integrated for the first time using for their numerical evaluation a GOSAM code, while the main contributions for the process $p p \rightarrow W^{+} W^{-}+2$ jets were already be computed in [21]. In [17] we included the computation of the closed fermion loops with electroweak gauge bosons attached and the non top resonant contribution form the third generation.

\section{Interfacing GOSAM}

Pointwise tests and integrations based on the re-weighting procedure for complicated two to four one loop processes show how our public package GOSAM can be efficiently used as a fully automatic generator for the virtual amplitudes. However, that is only a single piece in the task of the full automatic NLO code generation. The standard communication setup established in the BLHA gives then the possibility to link GOSAM to any other program that supports the same standard and supplement the other ingredients. The level of the automation in the generation of the tree level Born and real corrections, of the subtractions of the divergences as well as in the management of the integrations and the analysis of the results is already very high. We exploited 
the standard to link GOSAM to the SHERPA package that since the release 1.4 is equipped with all the ingredients needed for the parton level next to leading order simulation of QCD correction to the Standard Model processes, so that the whole NLO code is generated by a single commandline executable [22]. In this way the generation of the whole code is fully automatic so that once GOSAM provides the virtual amplitudes all the features already implemented in SHERPA for the leading order analyses become available for the NLO computation. Representative examples of distributions obtained with code generated fully automatically with GOSAM+SHERPA are given in Figures 2, 3 and 4 for the processes $p p \rightarrow W^{-} b \bar{b}$ with massive bottom quarks, $p p \rightarrow W^{-}+2$ jets and $p p \rightarrow W^{+} W^{+}+2$ jets respectively. The comparisons have beed done with MCFM [15] for the former two processes and with [23] for the latter. In conclusion we have presented the GOSAM
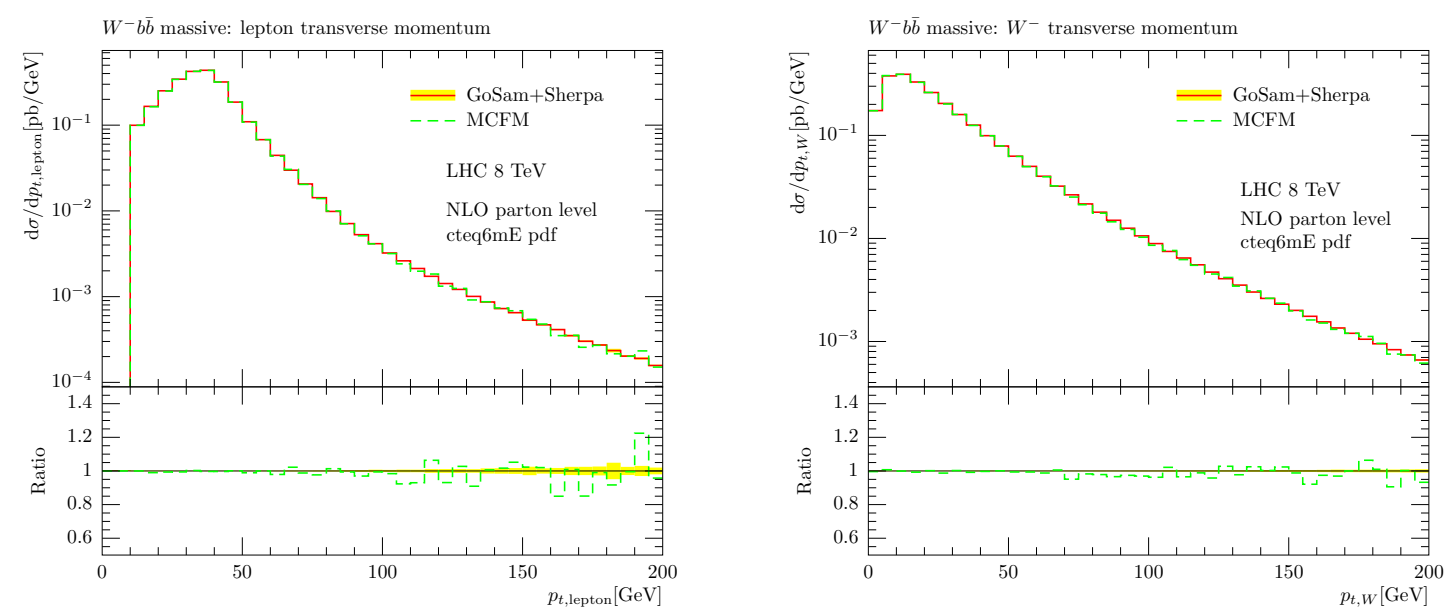

Figure 2: Charged lepton and $W^{-}$transverse momentum distribution for the process $p p \rightarrow W^{-} b \bar{b}$. Prediction obtained with GOSAM+SHERPA are compared to MCFM for a set of standard cuts.
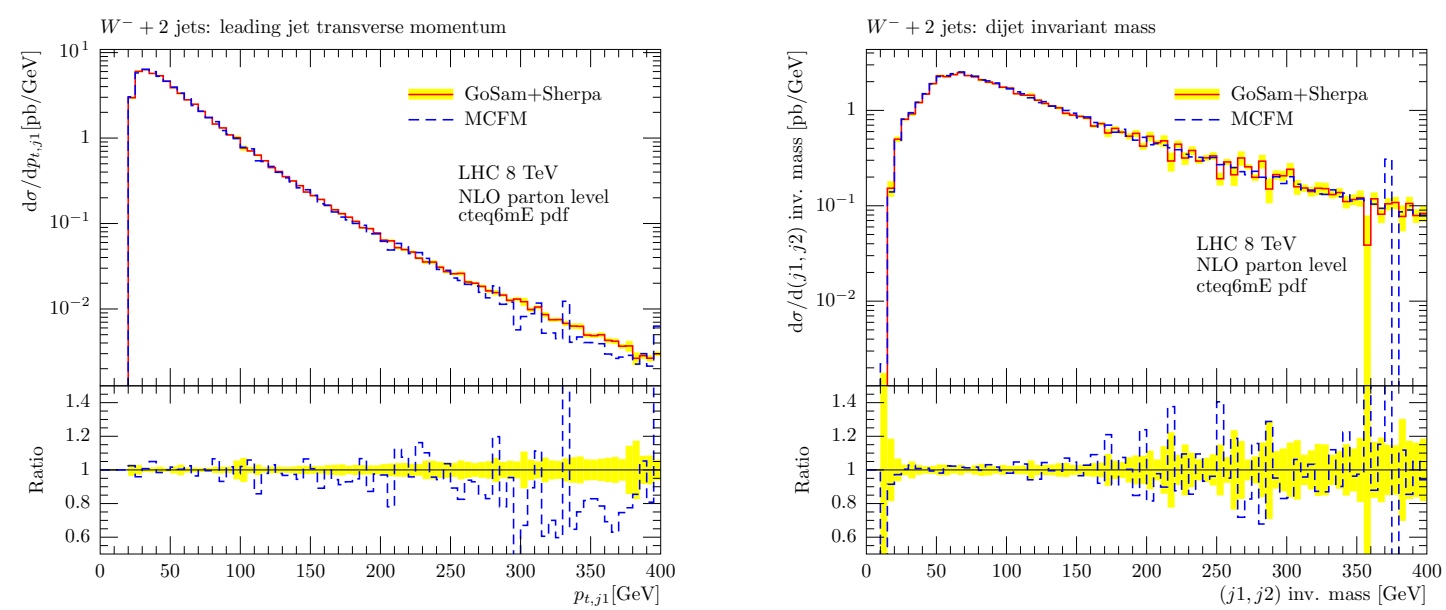

Figure 3: Leading jet transverse momentum and diet invariant mass distributions for the process $p p \rightarrow$ $W^{-}+2$ jets. Prediction obtained with GOSAM+SHERPA are compared to MCFM for a set of standard cuts.

package for the generation of numerical codes for the virtual amplitudes at one loop and shown 

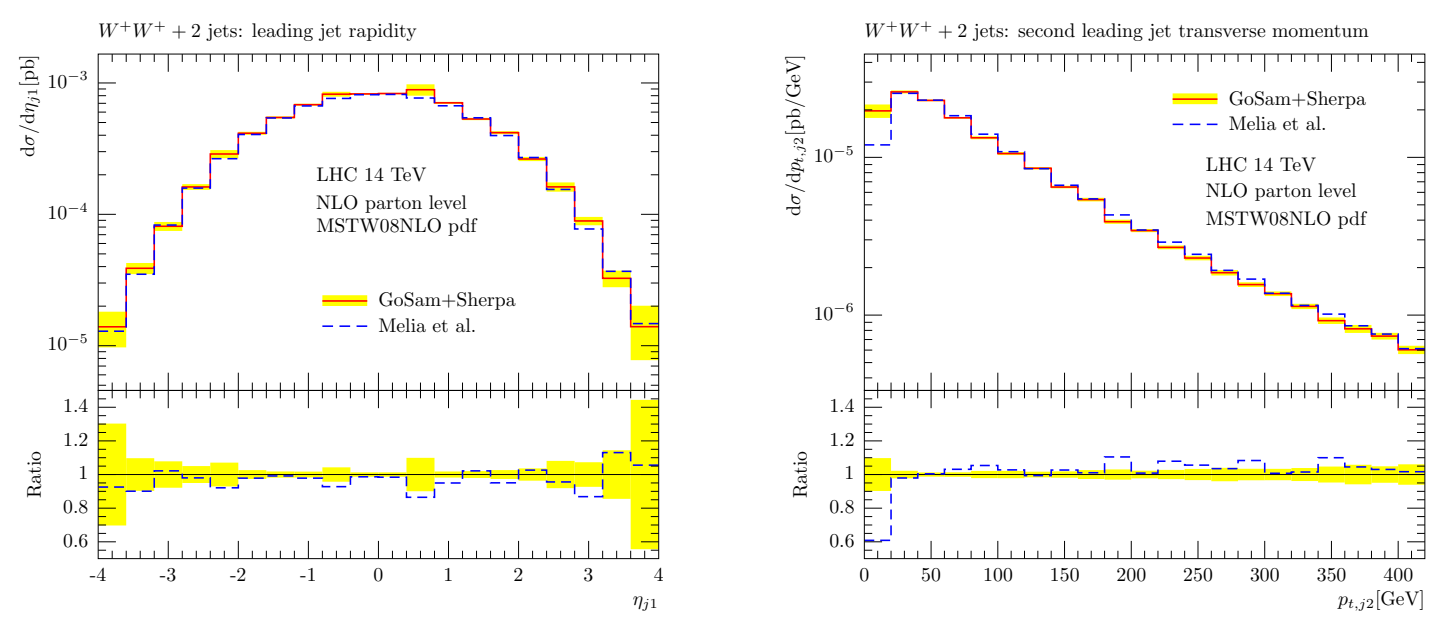

Figure 4: Rapidity of the leading jet and second jet transverse momentum distributions for the process $p p \rightarrow W^{+} W^{+}+2$ jets. Prediction obtained with GOSAM+SHERPA are compared with plots extracted from [23] following the phenomenological analysis reported there.

how the interface with other public codes can be exploited to have the fully automatic generation of programs for the computation of the NLO correction to the hard scattering processes.

\section{References}

[1] G. Aad et al. [ATLAS Collaboration], Phys. Lett. B 710 (2012) 49

S. Chatrchyan et al. [CMS Collaboration], Phys. Lett. B 710 (2012) 26

[2] M.L. Mangano, M. Moretti, F. Piccinini, R. Pittau, A.D. Polosa (2003) JHEP 0307001 (Preprint hep-ph/0206293)

T. Sjostrand, S. Mrenna, P.Z. Skands (2008) Comput.Phys.Commun. 178852 (Preprint 0710.3820)

G. Corcella, J.G. Knowles, G. Marchesini, S. Moretti, K. Odagiri, P. Richardson, M.H. Seymour, B.R. Webber (2001) JHEP 0101010 (Preprint hep-ph/0011363)

T. Gleisberg, S. .Hoeche, F. Krauss, M. Schonherr, S. Schumann, F. Siegert and J. Winter, JHEP 0902 (2009) 007

[3] S. Frixione, B.R. Webber (2002) JHEP 0206029 (Preprint hep-ph/0204244)

S. Frixione, F. Stoeckli, P. Torrielli, B.R. Webber, C.D. White (2010) (Preprint 1010.0819)

P. Nason (2004) JHEP 0411040 (Preprint hep-ph/0409146)

S. Frixione, P. Nason, C. Oleari (2007) JHEP 0711070 (Preprint 0709.2092)

S. Alioli, P. Nason, C. Oleari, E. Re (2010) JHEP 1006043 (Preprint 1002.2581)

S. Hoeche, F. Krauss, M. Schonherr and F. Siegert, [arXiv:1201.5882 [hep-ph]].

[4] T. Binoth, F. Boudjema, G. Dissertori, A. Lazopoulos, A. Denner, S. Dittmaier, R. Frederix and N. Greiner et al., Comput. Phys. Commun. 181 (2010) 1612

[5] G. Cullen, N. Greiner, G. Heinrich, G. Luisoni, P. Mastrolia, G. Ossola, T. Reiter and F. Tramontano, Eur. Phys. J. C 72 (2012) 1889

[6] P. Nogueira (1993) J.Comput.Phys. 105 279-289. 
[7] J. A. M. Vermaseren, math-ph/0010025.

[8] G. Cullen, M. Koch-Janusz, T. Reiter, Comput.Phys.Commun. 182, 2368 (2011).

[9] T. Reiter, Comput.Phys.Commun. 181, 1301 (2010).

[10] G. Cullen, J.P. Guillet, G. Heinrich, T. Kleinschmidt, E. Pilon, T. Reiter, M. Rodgers (2011) Comput.Phys.Commun. 1822276 (Preprint 1101.5595)

P. Mastrolia, G. Ossola, T. Reiter, F. Tramontano, JHEP 1008, 080 (2010).

R. K. Ellis and G. Zanderighi, JHEP 0802 (2008) 002

A. van Hameren, Comput. Phys. Commun. 182 (2011) 2427

[11] G. Passarino and M. J. G. Veltman, Nucl. Phys. B 160 (1979) 151.

[12] A. Denner, S. Dittmaier (2006) Nucl.Phys. B734 62 (Preprint hep-ph/0509141)

T. Binoth, J. P. .Guillet, G. Heinrich, E. Pilon and C. Schubert, JHEP 0510 (2005) 015

[13] G. Ossola, C.G. Papadopoulos, R. Pittau, Nucl.Phys. B763, 147 (2007).

R. Ellis, W.T. Giele, Z. Kunszt, K. Melnikov, Nucl.Phys. B822, 270 (2009).

[14] F. Cascioli, P. Maierhofer, S. Pozzorini, Phys.Rev.Lett. 108, 111601 (2012).

V. Hirschi, R. Frederix, S. Frixione, M.V. Garzelli, F. Maltoni et al. (2011) JHEP 1105044 (Preprint 1103.0621)

G. Bevilacqua, M. Czakon, M. V. Garzelli, A. van Hameren, A. Kardos, C. G. Papadopoulos,

R. Pittau and M. Worek, arXiv:1110.1499 [hep-ph].

A. van Hameren, JHEP 0907 (2009) 088

S. Badger, B. Biedermann, P. Uwer (2011) Comput.Phys.Commun. 1821674 (Preprint 1011.2900)

C. F. Berger, Z. Bern, L. J. Dixon, F. Febres Cordero, D. Forde, H. Ita, D. A. Kosower and D. Maitre, Phys. Rev. D 78 (2008) 036003

C. F. Berger, Z. Bern, L. J. Dixon, F. Febres Cordero, D. Forde, T. Gleisberg, H. Ita and

D. A. Kosower et al., Phys. Rev. Lett. 102 (2009) 222001

[15] J. M. Campbell and R. K. Ellis, Phys. Rev. D 60 (1999) 113006

[16] N. Greiner, A. Guffanti, T. Reiter and J. Reuter, Phys. Rev. Lett. 107 (2011) 102002

[17] N. Greiner, G. Heinrich, P. Mastrolia, G. Ossola, T. Reiter and F. Tramontano, Phys. Lett. B 713 (2012) 277

[18] T. Stelzer and W. F. Long, Comput. Phys. Commun. 81 (1994) 357

[19] R. Frederix, T. Gehrmann, N. Greiner (2008) JHEP 0809122 (Preprint 0808.2128)

R. Frederix, T. Gehrmann, N. Greiner (2010) JHEP 1006086 (Preprint 1004.2905)

[20] F. Maltoni and T. Stelzer, JHEP 0302 (2003) 027

[21] T. Melia, K. Melnikov, R. Rontsch and G. Zanderighi, Phys. Rev. D 83 (2011) 114043

[22] For download and installation details see the web page: http://gosam.hepforge.org/proc/

[23] T. Melia, K. Melnikov, R. Rontsch and G. Zanderighi, JHEP 1012 (2010) 053 